\title{
Theory of a Probe in a Strong Magnetic Field*
}

\author{
Juan R. Sanmartin $\dagger$ \\ Unwersity of Colorado, Boulder, Colorado and Plasma Physics Laboratory \\ Princeton University, Princeton, New Jersey \\ (Recerved 23 May 1968; final manuscript received 29 August 1969)
}

\begin{abstract}
An asymptotic analysis of the Langmuir-probe problem in a quiescent, fully ionized plasma in a strong magnetic field is performed, for electron cyclotron radius and Debye length much smaller than probe radius, and this not larger than either ion cyclotron radius or mean free path. It is found that the electric potential, which is not confined to a sheath, controls the diffusion far from the probe; inside the magnetic tube bounded by the probe cross section the potential overshonts to a large value before decaying to its value in the body of the plasma. The electron current is independent of the shape of the body along the field and increases with ion temperature; due to the overshoot in the potential, (1) the current at negative voltages does not vary exponentially, (2) its magnitude is strongly reduced by the field, and (3) the usual sharp knee at space potential, disappears. In the regions of the $C-V$ diagram studied the ion current is negligible or unaffected by the field. Some numerical results are presented. The theory, which fails beyond certain positive voltage, yields useful results for weak fields, too.
\end{abstract}

\section{INTRODUCTION}

The electrostatic probe is a very simple diagnostic tool used in the study of ionized gases. It is a small metallic electrode which is placed in the plasma at the location of interest; external circuitry is provided to vary its electric potential. The $C-V$ diagram or probe characteristic, which is a plot of the current flowing between the probe and the plasma versus the probe potential, may provide important information about the local properties of the plasma.

Since Langmuir's pioneer work, ${ }^{1}$ the theory of probes in the absence of magnetic fields has been extensively developed. In the collisionless limit (when $\lambda \gg R$ and $\lambda \gg \lambda_{D}$, where $\lambda, R$, and $\lambda_{D}$ are the mean-free-path, probe radius, and electron Debye length, respectively) the theory is practically complete and extensive computed results are available. ${ }^{2-5}$ The continuum case $\left(\lambda \ll \lambda_{D} \ll R\right)$ has been successfully treated by Su and $\mathrm{Lam}^{6}$ and Cohen. ${ }^{7}$ Recently, some attempts have been made to cover the intermediate regimes. ${ }^{8-11}$

* Partly based on a Ph.D. thesis presented to the University of Colorado in 1967.

$\dagger$ Present address: Mechanical Engineering Department, Massachusetts Institute of Technology, Cambridge, Massachusetts.

${ }^{1} \mathrm{I}$. Langmuir, in Collected Works of Irving Langmuir, C. G. Suits, Ed. (Pergamon Press, Inc., New York, 1961) Vols. 3,4 , and 5 .

2. I. B. Bernstein and I. Rabinowitz, Phys. Fluids 2, 112 $(1959)$.

S. H. Lam, Phys. Fluids 8, 73, 1002 (1965).

6. B. Bienkowski, Phys. Fluids 10, 675 (1967)

$5 \mathrm{~J}$. G. Laframboise, University of Toronto Institute for Aerospace Studies Report No. 100 (1966).

6. H. Su and S. H. Lam, Phys. Fluids 6, 1479 (1963).

${ }^{7}$ I. Cohen, Phys. Fluids $\sigma, 1492$ (1963).

${ }^{8}$ E. Wasserstrom, C. H. Su, and R. F. Probstein, Phys. Fluids $8,56(1965)$.

Y. S. Chou, L. Talbot, and D. R. Willis, Phys. Fluids 9, 2150 (1966).
The theory of probes in the presence of a magnetic field $\mathbf{B}$ is still in a primitive state. It is known experimentally that the field substantially modifies the $C-V$ diagram. The usual sharp knee at the plasma or space potential (the potential of the undisturbed plasma surrounding the probe) is blurred or disappears entirely; for more positive probe voltages (i.e., for electron collection) the current decreases substantially from its value for zero magnetic field. The theory confronts three basic difficulties. First, the presence of $\mathbf{B}$ makes the plasma anisotropic. Second, for large $B$, collisions come into play even if $\lambda$ is large compared with $R$. This leads to two effects. Firstly, the equations describing the problem in the neighborhood of the probe differ markedly from those valid far from the probe. Secondly, transport coefficients have to be considered and for fully ionized plasmas these coefficients will be spatially dependent because they depend on the charged particles densities.

Spivak and Reichrudel ${ }^{12}$ used orbital theory to consider the case of a weak magnetic field. Bickerton. and Von Engel ${ }^{13}$ and Nobata ${ }^{14}$ also used orbital theory to consider a plane probe, parallel to a strong magnetic field. They assumed that the electric field was confined to a sheath and no analysis was given to determine the density on the sheath edge. The potential field inside the sheath was assumed to have some definite shape.

${ }^{10} \mathrm{C} . \mathrm{H} . \mathrm{Su}$, in $A$ Survey of Phenomena in Ionized Gases (International Atomic Energy Agency, Vienna, 1968), p. 569.

$11 \mathrm{G}$. K. Bienkowski and K. W. Chang, Phys. Fluids 11, $784(1968)$.

12 G. Spivak and E. Reichrudel, Physik Z. Sowjetunion 9, $655(1936)$.

${ }_{13}$ R. J. Bickerton and A. von Engel, Proc. Phys. Soc. (London) B69, 468 (1956).

14 K. Nobata, J. Appl. Phys. (Japan) 2, 719 (1963). 
Bertotti ${ }^{16}$ studied a plane probe placed perpendicular to B. He averaged all variables over the probe cross section and assumed an unspecified diffusion process. A phenomenological integrodifferential equation was derived and numerically integrated. The results are in clear contradiction to all experimental evidence.

Bohm, ${ }^{16}$ who considered an arbitrarily shaped probe in a weakly ionized plasma in a magnetic field, assumed that the ion-to-electron temperature ratio was very small and that the electric field had a negligible effect on the electrons. He also assumed that the electrons moved freely across their last mean-free-path to the probe while at larger distances the plasma was collision dominated and a diffusion (Laplace's) equation for the electron density held. $\mathrm{He}$ then obtained an approximate expression for the electron current. The probe voltage was assumed to be near space potential but the dependence of the current on the probe potential was not given. (A correction by Sugawara ${ }^{17}$ tried to yield this dependence.)

In the present paper we consider a spherical probe and a quiescent, fully ionized plasma in the presence of a strong magnetic field. With the five characteristic lengths of the problem, $\lambda_{D}, R, \lambda, l_{s}$, and $l_{i}$ (where $l_{\text {e }}$ and $l_{i}$ are the electron and ion cyclotron radii), we define four nondimensional parameters,

$$
\begin{array}{rlrl}
\mu & =l_{e} / l_{i}, & & \sigma=l_{\odot} / R, \\
\epsilon=\lambda_{D} / R, & \tau & =R / \lambda,
\end{array}
$$

and perform an asymptotic analysis on the basis of the following assumptions:

(A) The electron-to-ion temperature ratio is $O(1)$. Because the electron-to-ion mass ratio is very small, this assumption implies $\mu<O(1)$ (i.e., $\mu \ll 1$ ).

(B) $\sigma<O(1)$. This may be considered as our definition of a strong magnetic field. We point out that the results of the analysis are found to be of use for weaker fields down to the limit $B=O(\sigma \rightarrow \infty)$; this is discussed in the last section.

(C) $\epsilon<O(1)$. This condition is often satisfied in actual plasmas and probes.

(D) $\tau \leq O(1)$, which means that the only case that we exclude is when $\lambda \ll R$.

(E) $\mu / \sigma \leq O(1)$.

Except for condition $(\mathrm{E})$, no relative order is

15 B. Bertotti, Phys. Fluids 4, 1047 (1961); 5, 1010 (1962).

10 D. Bohm, in The Characteristics of Electrical Discharges in Magnetic Fields, A. Guthrie and R. Ir. Wakerling, Eds. (McGraw-Hill Book Company, New York, 1949), Chaps. 2 and 3.

${ }_{17}$ M. Sugawara, Phys. Fluids 9, 797 (1966). imposed on the magnitudes of the parameters given above. Note, moreover, that

$$
\epsilon \tau \equiv \lambda_{D} / \lambda=O\left(\Lambda_{\infty}^{-1} \ln \Lambda_{\infty}\right),
$$

where $\Lambda_{\infty}$ is the ratio of $\lambda_{D}$ to impact parameter for single $90^{\circ}$ deflection. Thus, if $\Lambda_{\infty} \gg 1$, as is usually the case, condition (C) follows from (D) if $\tau=O(1)$.

The relatively weak conditions $(\mathrm{A})-(\mathrm{E})$ will allow us to obtain an expression for the electron component of the current to the probe. To determine the ion current (in the region of the $C-V$ diagram where it is important) we shall change conditions (D) and $(\mathrm{E})$ into the stronger conditions

$$
\begin{gathered}
\left(\mathrm{D}^{\prime}\right) \quad R<\lambda_{,} \\
\left(\mathrm{E}^{\prime}\right) \quad R<l_{i} .
\end{gathered}
$$

In the next section, the equations to be used are presented and nondimensional variables are defined. In Sec. III a nonlinear partial differential equation for the electric field is derived, whose solution yields the electron current reaching the probe. In Sec. IV an analysis is made of the probe characteristic. Two regions of the $C-V$ diagram are considered. In one region, around space potential, the ion component of the total current may be neglected. (For more positive voltages the ion current is even more negligible but the validity of our results breaks down beyond a certain probe voltage, which is determined.) In this region, therefore, the electron current computed here is approximately equal to the total current. The second region covers probe potentials highly negative with respect to space potential. We show that, under conditions (A)-( $\left.\mathrm{E}^{\prime}\right)$, the ion current in this region may be computed as if no magnetic field were present and therefore, known results for' this problem ${ }^{2-11}$ may be used. This region includes the ion saturation current and the floating potential (the probe potential for which no net current is collected).

In the last section we discuss some physical consequences of the analysis of Sec. III and the results of Sec. IV. Extensions of the theory are also discussed.

Finally we wish to point out that while conditions (A)-(E) are relatively weak, the equations to be used in the analysis impose a strong restriction on the application of the results: We use steady-state kinetic equations, and thus the possibility of anomalous transport processes due to fluctuating fields is excluded from the study; as a consequence only collisional transport coefficients are considered. The restrictions that this entails will be discussed in the last section, too. 


\section{BASIC EQUATIONS}

We consider a quiescent, fully ionized plasma in the presence of a static, uniform magnetic field $B$. A spherical probe of radius $R$ is located inside the plasma. The probe surface is perfectly absorbing and is at an electric potential $\phi_{p}$ (measured with respect to the undisturbed potential in the plasma). The basic equations governing the problem are the kinetic equation for the distribution function of the $a$ species, $F^{a}$,

$$
\begin{aligned}
& {\left[w_{z} \frac{\partial}{\partial z}+w_{r} \frac{\partial}{\partial r}-\frac{q_{a}}{m_{a}}\left(\frac{\partial V}{\partial z} \frac{\partial}{\partial w_{z}}+\frac{\partial V}{\partial r} \frac{\partial}{\partial r}\right)\right.} \\
& +\frac{q_{a} B}{m_{a} c}\left(w_{\theta} \frac{\partial}{\partial w_{r}}-w_{r} \frac{\partial}{\partial w_{\theta}}\right) \\
& \left.+\frac{w_{\theta}}{r}\left(w_{\theta} \frac{\partial}{\partial w_{r}}-w_{r} \frac{\partial}{\partial w_{\theta}}\right)\right] F^{a}=\sum_{b} C\left(F^{a}, F^{b}\right)
\end{aligned}
$$

and Poisson's equation for the potential, $V$,

$$
\nabla^{2} V=4 \pi e\left(\int F^{e} d \mathrm{w}-Z_{\imath} \int F^{i} d \mathrm{w}\right) ;
$$

$a$ and $b$ stand for $e$ or $i$ (electrons or ions, respectively), $q$ and $m$ are the particle charge and mass, $Z_{3}$ is the ion charge number $\left(q_{i}=Z_{i} e\right), c$ is the velocity of light, and $\mathbf{r} \equiv(z, r, \theta)$ and $\mathrm{w} \equiv\left(w_{z}\right.$, $w_{r}, w_{0}$ ) are the position and velocity vectors, respectively. We have used cylindrical coordinates centered in the probe with the $z$ axis along $\mathrm{B}$ so that $F^{a}$ and $V$ do not depend on $\theta$; the term $w_{\theta} / r\left(w_{\theta} \partial / \partial w_{r}\right.$ $\left.w_{r} \partial / \partial w_{\theta}\right) F^{a}$ in (1) is due to the non-Cartesian character of the coordinates.

$C\left(F^{a}, F^{b}\right)$ is the usual Fokker-Planck collision term $^{18}$ :

$$
\begin{gathered}
C\left(F^{a}, F^{b}\right)=\frac{2 q_{a}^{2} q_{b}^{2}}{m_{a}^{2}} \frac{\partial}{\partial \mathrm{w}} \cdot \iint d \mathrm{w}^{\prime} d \boldsymbol{\kappa} \frac{\boldsymbol{\kappa} \boldsymbol{\kappa}}{\kappa^{4}} \delta\left[\boldsymbol{k} \cdot\left(\mathrm{w}-\mathrm{w}^{\prime}\right)\right] \\
\cdot\left(F^{b} \frac{\partial F^{a}}{\partial \mathrm{w}}-\frac{m_{a}}{m_{b}} F^{a} \frac{\partial F^{b}}{\partial \mathrm{w}^{\prime}}\right)
\end{gathered}
$$

where the usual cutoffs at small and large impact parameter, $\kappa^{-1}$, have to be introduced in the $k$ integral. The precise form (3) for $C$ will only be required for the computation of some transport coefficients in a spatial region where collisions dominate; in other parts of the analysis only the order of magnitude of the mean free path associated with $C$ will be needed. For either consideration the results of the Fokker-Planck and the more exact Balescu-Lenard collision operators are very similar. ${ }^{18}$ The use of (3) is discussed further in the next section.

${ }^{18}$ I. P. Shkarofsky, T. W. Johnston, and M. P. Bachinsky, The Particle Kinetics of Plasmas (Addison-Wesley Publishing Company, Reading, Massachusetts, 1966), Chaps. 7 and 8.
The boundary conditions for Eqqs. (1) and (2) are: On the probe

$$
V=V_{p}, \quad F^{a}=0 \text { for outgoing particles. }
$$

Far away from the probe

$$
\begin{aligned}
& V \rightarrow 0, \\
& \int F^{a} d \mathrm{w} \rightarrow N_{a \infty}, \quad \int F^{a} m_{a} \mathrm{~W}^{2} d \mathrm{w} \rightarrow 3 N_{a \infty} k T_{a \infty},
\end{aligned}
$$

and $F^{a}$ is an even function of w.

We now define

$f^{a}=F^{a} / N_{a \infty}\left(k T_{a \infty} / m_{a}\right)^{3 / 2}, \quad \mathrm{v}=\mathrm{w}\left(k T_{a \omega} / m_{a}\right)^{-1 / 2}$,

$\chi=e V\left(k T_{e \infty}\right)^{-1}, \quad \beta=T_{i \infty}\left(Z_{i} T_{\circ \infty}\right)^{-1}, n^{a}=\int f^{a} d \mathrm{v}$,

$D_{z}=v_{z} \frac{\partial}{\partial z}+\frac{\partial \chi}{\partial z} \frac{\partial}{\partial v_{z}}, \quad D_{z}^{i}=v_{z} \frac{\partial}{\partial z}-\frac{1}{\beta} \frac{\partial \chi}{\partial z} \frac{\partial}{\partial v_{z}}$,

$D_{r}=v_{\perp} \frac{\partial}{\partial r}+\frac{\partial \chi}{\partial r} \frac{\partial}{\partial v_{\perp}}, \quad D_{r}^{i}=v_{\perp} \frac{\partial}{\partial r}-\frac{1}{\beta} \frac{\partial \chi}{\partial r} \frac{\partial}{\partial v_{\perp}}$,

where $v_{\perp}=\left(v_{r}^{2}+v_{\theta}^{2}\right)^{1 / 2} ;$ also tn $\phi=v_{0} / v_{r}$.

Equation (1) now reads for $a=e$ and $a=i$, respectively,

$$
\begin{aligned}
& {\left[\frac{\partial}{\partial \phi}+l_{e} D_{z}+l_{e} \cos \phi D_{r}-l_{e} \sin \phi\right.} \\
& \left.\quad\left(\frac{v_{\perp}}{r}+\frac{1}{v_{\perp}} \frac{\partial \chi}{\partial r}\right) \frac{\partial}{\partial \phi}\right] f^{e}=\frac{l_{e}}{\lambda}\left[c\left(f^{e}, f^{e}\right)+c\left(f^{e}, f^{i}\right)\right],
\end{aligned}
$$

$\left[-\frac{l_{e}}{l_{i}} \frac{\partial}{\partial \phi}+l_{e} D_{z}^{i}+l_{\mathrm{o}} \cos \phi D_{r}^{2}-l_{e} \sin \phi\right.$

$$
\left.\cdot\left(\frac{v_{\perp}}{r}-\frac{\beta^{-1}}{v_{\perp}} \frac{\partial \chi}{\partial r}\right) \frac{\partial}{\partial \phi}\right] f^{i}=\frac{l_{\theta}}{\lambda}\left[c\left(f^{i}, f^{\prime}\right)+c\left(f^{i}, f^{o}\right)\right] .
$$

Equation (2) reads

$$
\lambda_{D}^{2}\left(\frac{\partial^{2}}{\partial z^{2}}+r^{-1} \frac{\partial}{\partial r} r \frac{\partial}{\partial r}\right) \chi=n^{0}-n^{i} .
$$

In Eqs. (8)-(10),

$$
\begin{gathered}
l_{a}=\frac{m_{a} c}{\left|q_{a}\right| B}\left(\frac{k T_{a \infty}}{m_{a}}\right)^{1 / 2}, \quad \lambda_{D}=\left(\frac{k T_{o}}{4 \pi N_{o a} e^{2}}\right)^{1 / 2}, \\
\lambda=\frac{\left(k T_{e \infty}\right)^{2}}{2 \pi N_{o \infty} e^{4} \ln \Lambda_{\infty}}, \\
\Lambda_{\infty}=\frac{3 k T_{e \infty}}{e^{2}}\left(\frac{k T_{o \infty}}{4 \pi N_{\infty \infty} e^{2}\left(1+\beta^{-1}\right)}\right)^{1 / 2}, \\
\lambda^{-1} \sum_{b} c\left(f^{a}, f^{b}\right)=\frac{k T_{a \infty}}{m_{a} N_{a \infty}} \sum_{b} C\left(F^{a}, F^{b}\right),
\end{gathered}
$$

where $l_{a}$ is the cyclotron radius of the $a$ species, $\lambda_{D}$ is the electron Debye length, $\lambda$ is the mean-freepath (electron scattering distance for Coulomb 
collisions), $\Lambda_{\infty}$ is the ratio of $\lambda_{D}$ to impact parameter for single $90^{\circ}$ deflections and $c\left(f^{a}, f^{b}\right)$ is a nondimensional form of the collision term; an arbitrary factor of order unity could enter into the definition of $\lambda$.

In nondimensional variables the boundary conditions become: On the probe

$$
\chi=\chi_{p}, f^{a}=0 \text { for outgoing particles. }
$$

Far away from the probe

$$
x \rightarrow 0, \quad n^{a} \rightarrow 1, \quad \int f^{a} v^{2} d v \rightarrow 3,
$$

and $f^{a}$ is even in $\mathrm{v}$.

We now define the parameters $\mu=l_{e} / l_{\imath}, \sigma=$ $l_{e} / R, \epsilon=\lambda_{D} / R$, and $\tau=R / \lambda$ and introduce assumptions $(\mathrm{A})-(\mathrm{E})$ given in Sec. I. Whenever the electron and ion densities and temperatures are of the same order of magnitude as $N_{\epsilon \infty}, T_{\epsilon \infty}$, and $N_{2 \infty}, T_{2 \infty}$ respectively, condition (A) [i.e., $\beta=O(1)$ ] implies that $c\left(f^{a}, f^{b}\right)=O(1)$ [for $\left.|\mathrm{v}|=O(1)\right]$ for any $a$ and $b$. Also every term on the left-hand side of (8) or (9) is now the ratio of two characteristic lengths, times a factor of $O(1)$. Finally, we may write

$$
c\left(f^{e}, f^{i}\right)=n^{i} P\left(f^{e}\right)+\mu P_{\mu}\left(f^{e}, f^{2}\right),
$$

where $P_{\mu}\left(f^{e}, f^{2}\right)=O(1)$ and

$$
\begin{array}{r}
P\left(f^{0}\right)=\frac{A}{v^{3}}\left(\frac{\partial}{\partial \nu}\left(1-\nu^{2}\right) \frac{\partial}{\partial \nu}+\left(1-\nu^{2}\right)^{-1} \frac{\partial^{2}}{\partial \phi^{2}}\right) f^{\theta}, \\
\left(\nu=\frac{v_{z}}{v}\right)
\end{array}
$$

and $A=Z_{1} \ln \Lambda / \ln \Lambda_{\infty}\left[\Lambda=\Lambda_{\infty}\left(n^{e}\right)^{-1 / 2}\right.$ if the local temperatures are the same as the temperatures far away from the probe]. ${ }^{18}$ Equation (16) is easily derived from (3) and (13). Note that if $f^{e}$ is isotropic in $\mathrm{v}, P\left(f^{e}\right)=0$. The decomposition in (16) reflects the fact that to zero order in $\mu$, ion velocities are negligible when compared with electron velocities. Equations (8)-(10) are now ready for an asymptotic analysis.

\section{THE EQUATION FOR THE ELECTRON CURRENT}

Consider the present problem and assume that $B=0$. Since the problem now has spherical symmetry the directed flux toward the probe decays as $\left(r^{2}+z^{2}\right)^{-1}$, and this results in immediate first integrals of the electron and ion continuity equations. If the probe is small, this geometrical spreading of the current makes probe perturbations negligible at distances still "microscopic."

When a magnetic field is present, no such first integrals are available. The continuity equations are not eliminated, and they will clearly be of foremost importance. For large $B$, the electrons follow closely the lines of force. On the plane $z=0$, outside the probe, the $z$ component of the flux is zero by sym. metry, while it cannot vanish on the probe surface. Hence, strong gradients will develop around the boundary of the "shadow," the tube of force incident on the probe cross section. As one travels away from the probe, the gradients will be smoothed by collisions that draw electrons into the shadow. Since collisional diffusion across a strong magnetic field is very small, probe perturbations will die out very slowly along B. The emphasis on the continuity equation and the channeling of probe perturbations over large distances along $\mathbf{B}$ are the first outstanding effects of the magnetic field.

\section{A. The Outer $z$ Region}

Consider the electron continuity equation

$$
\frac{1}{r} \frac{\partial}{\partial r} r j_{r}^{e}+\frac{\partial}{\partial z} j_{z}^{e}=0
$$

where $j_{z}^{a}$ and $j_{r}^{s}$ are the components of the electron flux corresponding to the $z$ and $r$ coordinates, respectively. Obviously, $r^{-1} \partial / \partial r r=O\left(R^{-1}\right)$ and from a known result for the collisional diffusion coefficient acioss a strong magnetic field $\left(l_{0} \ll \lambda\right){ }^{18}$ we also have $j_{r}^{s}=O\left(l_{e}^{2} \lambda^{-1} R^{-1}\right)$. If $L_{0}$ is the characteristic length of the extent along $B$ of the perturbations caused by the probe, there follows from (18)

$$
j_{z}^{e}=O\left(\frac{l_{\theta}^{2} L_{0}}{R^{2} \lambda}\right) .
$$

On the other hand, from the value of the collisional diffusion coefficient along a magnetic field we have $j_{z}^{e}=O\left(\lambda / L_{0}\right)$. Hence,

$$
L_{0}=\frac{R \lambda}{l_{s}}, \quad j_{2}^{e}=O(\sigma) .
$$

Therefore, we introduce the nondimensional variables

$$
r_{0}=r / R, \quad z_{0}=z l_{o} / R \lambda .
$$

The condition of no perturbations far away from the probe, (15), has to be satisfied at infinity in the $\left(r_{0}, z_{0}\right)$ variables. On the other hand, we may not rule out the need for a new length scaling near the probe if the solution resulting from the use of scaling (19) cannot satisfy the boundary conditions on the probe. The use of several length scalings is standard technique in boundary-layer analysis in fluid mechanics. Here, $r_{0}$ and $z_{0}$ will be called outer variables.

Using (19), Eqs. (8)-(10) become 


$$
\begin{gathered}
{\left[\frac{\partial}{\partial \phi}+\sigma \cos \phi D_{r_{0}}-\sigma \sin \phi\left(\frac{v_{\perp}}{r_{0}}+\frac{1}{v_{\perp}} \frac{\partial \chi}{\partial r_{0}}\right)\right.} \\
\left.\cdot \frac{\partial}{\partial \phi}+\sigma^{2} \tau D_{z_{0}}\right] f^{e} \\
=\sigma \tau\left[c\left(f^{e}, f^{e}\right)+n^{i} P\left(f^{e}\right)+\mu P_{\mu}\left(f^{e}, f^{i}\right)\right] \\
{\left[\sigma \cos \phi D_{r_{0}}^{i}-\sigma \sin \phi\left(\frac{v_{\perp}}{r_{0}}-\frac{\beta^{-1}}{v_{\perp}} \frac{\partial \chi}{\partial r_{0}}\right)\right.} \\
\left.\cdot \frac{\partial}{\partial \phi}-\mu \frac{\partial}{\partial \phi}+\sigma^{2} \tau D_{z \circ}\right] f^{i} \\
=\sigma \tau\left[c\left(f^{i}, f^{i}\right)+c\left(f^{i}, f^{o}\right)\right], \\
\epsilon^{2}\left(\frac{1}{r_{0}} \frac{\partial}{\partial r_{0}} r_{0} \frac{\partial}{\partial r_{0}}+\sigma^{2} \tau^{2} \frac{\partial^{2}}{\partial z_{0}^{2}}\right) \chi=n^{e}-n^{i} .
\end{gathered}
$$

Next, we introduce the expansions

$$
\begin{aligned}
& f^{e}=f_{0}^{e}+\delta_{1} f_{1}^{e}+\delta_{2} f_{2}^{e}+\cdots, \\
& f^{i}=f_{0}^{i}+\delta_{1}^{i} f_{1}^{i}+\cdots, \\
& \chi=\chi_{0}+\Delta_{1} \chi_{1}+\cdots,
\end{aligned}
$$

and study Eqs. (20)-(22) successively. From now on we shall substitute $\chi_{0}$ for $\chi$ in (6) and (7) unless otherwise stated.

To zero order, Eq. (20) yields $\partial f_{0}^{\circ} / \partial \phi=0$. To the next order

$\delta_{1} \frac{\partial f_{1}^{e}}{\partial \phi}+\sigma \cos \phi D_{r_{0}} f_{0}^{e}=\sigma \tau\left[c\left(f_{0}^{e}, f_{0}^{e}\right)+n_{0}^{i} P\left(f_{0}^{e}\right)\right]$.

The right-hand side of (24) is $\phi$ independent; since $f_{1}^{e}$ has to be periodic in $\phi$, we must have

$$
\begin{aligned}
\delta_{1} \frac{\partial f_{1}^{e}}{\partial \phi}+\sigma \cos \phi D_{r_{0}} f_{0}^{e} & =0, \\
c\left(f_{0}^{e}, f_{0}^{e}\right)+n_{0}^{i} P\left(f_{0}^{e}\right) & =0 .
\end{aligned}
$$

Equation (25) gives

$$
\delta_{1} f_{1}^{e}=-\sigma \sin \phi D_{\mathrm{r}_{0}} f_{0}^{o}+\delta_{1 \mathrm{co}} f_{1 \mathrm{c}}^{i},
$$

where $\delta_{1 c} f_{i c}^{0}$ is $\phi$ independent; we observe that $f_{i}^{o}$ yields no radial flux. Equation (26) has as a unique solution a (local) Maxwellian distribution (see Appendix A); hence, $T_{\bullet \|}=T_{\epsilon \perp}$. Since the electric field is small in this outer region (collisions are dominant), the heating of the plasma can be neglected to zero order. Thus, $T_{0}=T_{\text {cos }}$ and

$$
f_{0}^{a}=n_{0}^{e} f_{m} \equiv n_{0}^{e}(2 \pi)^{-3 / 2} \exp \left(-v^{2} / 2\right),
$$

where $n_{0}^{\mathrm{a}}\left(r_{0}, z_{0}\right)$ is unknown $\left(n_{0}^{\circ} \rightarrow 1\right.$ at infinity).

To the next order, Eq. (20) yields

$$
\begin{aligned}
\delta_{2} \frac{\partial f_{2}^{e}}{\partial \phi} & +\sigma \delta_{1} \cos \phi D_{r_{0}} f_{1}^{e}+\sigma \Delta_{1} \cos \phi \frac{\partial \chi_{1}}{\partial r_{0}} \frac{\partial f_{0}^{e}}{\partial v_{\perp}} \\
& -\sigma \delta_{1} \sin \phi\left(\frac{v_{\perp}}{r_{0}}+\frac{1}{v_{\perp}} \frac{\partial \chi_{0}}{\partial r_{0}}\right) \frac{\partial f_{1}^{e}}{\partial \phi}+\sigma^{2} \tau D_{z_{0}} f_{0}^{e} \\
= & \sigma \tau\left[\delta_{1} c\left(f_{0}^{e}, f_{1}^{e}\right)+\delta_{1} c\left(f_{1}^{e}, f_{0}^{e}\right)\right. \\
& \left.+\delta_{1}^{i} n_{1}^{i} P\left(f_{0}^{e}\right)+\delta_{1} n_{0}^{i} P\left(f_{1}^{e}\right)+\mu P_{\mu}\left(f_{0}^{e}, f_{0}^{i}\right)\right] .
\end{aligned}
$$

The third term inside the bracket vanishes because $f_{0}^{*}$ is isotropic. We may also drop the term $\mu P_{\mu}\left(f_{0}^{0}, f_{0}^{i}\right)$ : it will be shown below that $f_{0}^{i}$ is a quiescent Maxwellian distribution and a simple analysis of Eqs. (3) and (13) shows that then $c\left(f_{0}^{e}, f_{0}^{i}\right)=O\left(\mu^{2}\right)$ and thus $\mu P_{\mu}=O\left(\mu^{2}\right)$ [and not $O(\mu)$ ].

If we finally substitute expression (27) for $\delta_{1} f_{1}^{0}$ in (29) we find that there are terms periodic in $\phi$ and $\phi$ independent terms in this equation. Since $f_{2}^{\circ}$ must be periodic, we may split (29) into two equations

$$
\begin{aligned}
& \delta_{2} \frac{\partial f_{2}^{e}}{\partial \phi}+\sigma \delta_{1} \cos \phi D_{r_{0}} f_{1}^{e} \\
& \quad+\sigma \Delta_{1} \cos \phi \frac{\partial \chi_{1}}{\partial r_{0}} \frac{\partial f_{0}^{e}}{\partial v}-\sigma \delta_{1} \cos \phi\left(\frac{v_{\perp}}{r_{0}}+\frac{1}{v_{\perp}} \frac{\partial \chi_{1}}{\partial r_{0}}\right) \frac{\partial f_{1}^{e}}{\partial \phi} \\
& =-\sigma^{2} \tau\left[c\left(f_{0}^{e}, \sin \phi D_{r_{0}} f_{0}^{e}\right)\right. \\
& \left.\quad+c\left(\sin \phi D_{r_{0}} f_{0}^{e}, f_{0}^{e}\right)+n_{0}^{i} P\left(\sin \phi D_{r_{0}} f_{0}^{e}\right)\right] \\
& \sigma^{2} \tau D_{z_{0}} f_{0}^{e}=\sigma \tau \delta_{1 o}\left[c\left(f_{0}^{e}, f_{10}^{e}\right)+c\left(f_{10}^{e}, f_{0}^{e}\right)_{\perp}+n_{0}^{i} P\left(f_{1 o}^{e}\right)\right] .
\end{aligned}
$$

Equation (31) yields $\delta_{10}=\sigma$; after f $f_{10}^{e}$ has been found we may compute $j_{z}^{e}$ :

$$
j_{z}^{e} \approx \delta_{1} j_{z 1}^{e}=\delta_{1 o} \int f_{10}^{e} v_{z} d v
$$

Equation (30) may easily be solved for $f_{2}^{e}$. However, we shall only be interested in the radial flux $j_{r}^{\circ} \approx$ $\delta_{2} j_{r 2}^{e}=\delta_{2} \int f_{2}^{e} v_{\perp} \cos \phi d v$. Note that only the righthand side of (30) contributes to this integral. Moreover, only the last term on the right can contribute to $j_{r_{2}}^{e}$ because there may be no self-acceleration of the electrons. Therefore,

$$
\delta_{2} j_{r 2}^{i}=\sigma^{2} \tau n_{0}^{i} \int P\left(\sin \phi D_{r_{0}} f_{0}^{e}\right) v_{\perp} \sin \phi d \mathrm{v} .
$$

If a relation between $\chi_{0}$ and $n_{0}^{\circ}$ is known, (32) and (33) give the electron flux as a function of $\chi_{\eta}$. If we now integrate (20) over $\mathrm{v}$, we obtain the electron continuity equation (18) which to dominant order reads

$$
\sigma^{3} \tau\left(\frac{1}{r_{0}} \frac{\partial}{\partial r_{0}} r_{0} j_{r 2}^{a}+\frac{\partial}{\partial z_{0}} j_{z 1}^{a}\right)=0 .
$$

This will be then an equation for $\chi_{0}$. 
To obtain a relation between $n_{0}^{\circ}$ and $\chi_{0}$, we consider Eqs. (21) and (22). This last equation yields

$$
n_{0}^{s}=n_{0}^{i} \text { (quasineutrality condition), }
$$

while to dominant order (21) becomes

$$
\begin{gathered}
\sigma \cos \phi D_{r_{0}}^{i} f_{0}^{i}-\sigma \sin \phi\left(\frac{v_{\perp}}{r_{0}}-\frac{\beta^{-1}}{v_{\perp}} \frac{\partial \chi_{0}}{\partial r_{0}}\right) \frac{\partial f_{0}^{i}}{\partial \phi}-\mu \frac{\partial f_{0}^{i}}{\partial \phi} \\
=\sigma \tau\left[c\left(f_{0}^{i}, f_{0}^{i}\right)+c\left(f_{0}^{i}, f_{0}^{e}\right)\right] .
\end{gathered}
$$

A simple analysis of Eqs. (3) and (13) shows that when $f_{0}^{e}$ is a Maxwellian distribution, $c\left(f_{0}^{i}, f_{0}^{e}\right)=O(\mu)$ and therefore, we may drop the last term on the right-hand side of (36). This equation may be rewritten as

$$
\begin{array}{r}
\sigma\left(\mathrm{v}_{\perp} \cdot \frac{\partial f_{0}^{i}}{\partial \mathbf{r}_{\perp 0}}-\beta^{-1} \frac{\partial \chi_{0}}{\partial \mathbf{r}_{\perp 0}} \cdot \frac{\partial f_{0}^{i}}{\partial \mathrm{v}_{\perp}}\right)+\mu \mathrm{v}_{\perp} \times \hat{z} \cdot \frac{\partial f_{0}}{\partial \mathrm{v}_{\perp}} \\
=\sigma \tau c\left(f_{0}^{i}, f_{0}^{i}\right),
\end{array}
$$

where $\hat{z}$ is a unit vector along the $z$ axis and $r_{\perp 0}$ and $v$ are Cartesian coordinates: $r_{\perp 0}=\left(r_{0} \cos \theta, r_{0} \sin \theta\right)$, $\mathrm{v}_{\perp}\left(v_{r} \cos \theta-v_{\theta} \sin \theta, v_{r} \sin \theta+v_{\theta} \cos \theta\right)$.

We show in Appendix A that the unique solution of Eq. (37) is

$$
\begin{aligned}
& f_{0}^{i}=K \exp \left(-\beta^{-1} \chi_{0}\right)(2 \pi)^{-3 / 2} \\
& \cdot \exp \left[-\frac{1}{2} \mathrm{v}_{\perp}^{2}-\frac{1}{2}\left(v_{z}-u_{z}\right)^{2}\right]
\end{aligned}
$$

where $K$ and $u_{z}$ are arbitrary functions of $z_{0}$. If we take the limit $r_{0} \rightarrow \infty$ with fixed $z_{0}$, we see that $K \equiv 1, u_{z} \equiv 0$. Thus, $f_{0}^{i}$ is a Maxwell-Boltzmann distribution (while $f_{0}^{e}$ is only locally Maxwellian).

From Eqs. (35) and (38) we see that

$$
n_{0}^{0}=\exp \left(-\beta^{-1} \chi_{0}\right) \text {. }
$$

When (39) is introduced into (32), the resulting equation is a nondimensional form of the equation used to compute the electric conductivity. Writing Spitzer's result for the conductivity ${ }^{19}$ in nondimensional form we obtain

$$
\delta_{1} \dot{j}_{z 1}^{s}=\sigma \frac{\gamma\left(Z_{i}\right)}{Z_{i}} \frac{\beta+1}{\beta}\left(\frac{2^{7}}{\pi}\right)^{1 / 2} \frac{\ln \Lambda_{\infty}}{\ln \Lambda} \frac{\partial \chi_{0}}{\partial z_{0}},
$$

where $\gamma\left(Z_{i}\right)$ is Spitzer's factor $[\gamma(1)=0.582, \gamma(\infty)=$ 1] and

$$
\Lambda=\Lambda_{\infty}\left(n_{0}^{e}\right)^{-1 / 2} \text {. }
$$

Introducing (39) into Eq. (33) and performing the integration there results

$$
\delta_{2} j_{r_{2}}^{o}=\sigma^{2} \tau Z_{i} \frac{\beta+1}{\beta} \frac{2^{3 / 2}}{3 \pi^{1 / 2}} \frac{\ln \Lambda}{\ln \Lambda_{\infty}} \exp \left(-2 \beta^{-1} \chi_{0}\right) \frac{\partial \chi_{0}}{\partial r_{0}},
$$

${ }^{19}$ L. Spitzer, Jr., Physics of Fully Ionized Gases (Interscience Publishers, Ine., New York, 1956), Chap. 5. which is a nondimensional form of the known diffusion coefficient transverse to a strong magnetic field. ${ }^{18}$

The use of the Folkker-Planck collision term is valid only if the Debye length is smaller than both the electron cyclotron radius and the characteristic length for macroscopic variations. The second condition is satisfied here but we have made no assumption on the ratio $\lambda_{D} / l_{\theta}$. Making the assumption $\lambda_{D} / l_{\mathrm{e}}<1$ would not solve the difficulty because the local Debye length has to be used in the comparison to $l_{e}$, and the Debye length increases as $n_{0}^{\circ}$ decreases. The consequence is that the use of a properly modified Fokker-Planck term would result in a definition of $\Lambda$ not so simple as that given in (41).

Here, we shall use an average value of $\ln \Lambda$, $(\ln \Lambda)$. One reason is that the results do not depend on $(\ln \Lambda)$ as we shall see immediately. A second reason is that the exponential term in (42) is the dominant effect in the spatial variation of the transport coefficients, while the variation in $\ln \Lambda$ is small for large $\ln \Lambda_{\infty}$; a further discussion on this is given in Sec. V. Finally, there is, at present, an imperfect knowledge of transport coefficients under conditions such that $l_{0}$ is smaller than the local Debye length.

We now introduce the new variables

$$
\psi=\frac{2 \chi_{0}}{\beta}, \quad x=r_{0}, \quad y=z_{0} \frac{Z_{i}}{(12 \gamma)^{1 / 2}} \frac{(\ln \Lambda)}{\ln \Lambda_{\infty}}
$$

into (34), (40), and (42) to obtain

$$
\begin{gathered}
\frac{\partial^{2} \psi}{\partial y^{2}}+\frac{1}{x} \frac{\partial}{\partial x} x \exp (-\psi) \frac{\partial \psi}{\partial x}=0 \\
\delta_{1} j_{z 1}^{s}=\sigma(\beta+1)\left(\frac{8 \gamma}{3 \pi}\right)^{1 / 2} \frac{\partial \psi}{\partial y}
\end{gathered}
$$

The elliptic equation (43) has to be solved under the condition

$$
\psi \rightarrow 0 \text { as }\left(x^{2}+y^{2}\right)^{1 / 2} \rightarrow \infty .
$$

We need a condition for $\psi$ about the probe, too. However, near the probe the expression for $f^{e}$ has to break down because on the probe surface there are no outgoing particles and $f_{0}^{a}$ cannot be isotropic. The probe surface is given by

$$
y=\frac{Z_{i}}{(12 \gamma)^{1 / 2}} \frac{(\ln \Lambda)}{\ln \Lambda_{\infty}} \sigma \tau\left(1-x^{2}\right)^{1 / 2}, \quad x \leq 1
$$

and therefore, the expansion for $f^{\circ}$ breaks down as $y \rightarrow 0$. We notice that the breakdown occurs for $x \lesssim 1$ only. Therefore, inside the shadow $(r \leq R)$, there is an inner layer where collisions will not be dominant. This implies that in this layer the gradients will be stronger than in the (outer) $z_{0}$ layer; a new scaling has to be used. The characteristic 
length of this inner $z$ layer, $\tilde{L}$, will be small compared with $R \lambda / l_{s}$. Therefore, within an error or order of $\left(\tilde{L} l_{s}\right)(R \lambda)^{-1}$, the electron flux along $\mathrm{B}$ is conserved in the inner layer. Outside the shadow, the flux reaching the plane $z=0$ has to vanish by symmetry and thus

$$
\frac{\partial \psi}{\partial y}=0, \quad \text { at } \quad y=0, \quad(x>1)
$$

because $j_{z 1}^{e} \sim \partial \psi / \partial y$ in the outer region, as given in (44). For $x \leq 1$, the expression (44) for $j_{z}^{c}$ has to be set equal (at every value of $x$ ) to the current density reaching the probe. This will result in a relation of the type

$$
Q\left(\psi, \frac{\partial \psi}{\partial y}\right)=0, \quad \text { at } \quad y=0, \quad(x \leq 1) .
$$

We need to determine this relation. If this is known, conditions (45)-(47) are sufficient to solve (43) in the half-plane $y \geq 0$. (From now on we shall consider the positive $z$ half-plane.)

\section{B. The First Inner Layer}

To satisfy the conditions on the probe we define (for $r_{0} \leq 1$ ) an inner variable $\tilde{z}=z / L$, where $L$ is such that $D_{\tilde{z}} f^{\theta}$ is now comparable to the collision term in (8). Next, we introduce into (8) the expansions $(23 \mathrm{~b})$ and

$$
\begin{aligned}
& f^{e}=\tilde{n}\left(\tilde{f}_{0}^{o}+\tilde{\delta}_{1} \tilde{f}_{1}^{o}+\cdots\right), \\
& \quad \chi=\ln \tilde{n}^{-\beta}+\tilde{\chi}_{0}+\tilde{\Delta}_{1} \tilde{\chi}_{1}+\cdots,
\end{aligned}
$$

where

$\tilde{n}\left(r_{0}\right)=\lim _{z_{0} \rightarrow 0} n_{0}^{e}=\lim _{z_{0} \rightarrow 0} \exp \left(-\beta^{-1} \chi_{0}\right), \quad\left(r_{0} \leq 1\right)$.

To zero order the resulting equation yields $\partial \tilde{f}_{0}^{\theta} / \partial \phi=0$ and to first order

$$
\begin{aligned}
& \tilde{n} \tilde{\delta}_{1} \frac{\partial \tilde{f}_{1}^{o}}{\partial \phi}+ \sigma \cos \phi\left(v_{\perp} \frac{\partial}{\partial r_{0}}\right. \\
&\left.+\frac{\partial}{\partial r_{0}}\left(\ln \tilde{n}^{-\beta}+\tilde{\chi}_{0}\right) \frac{\partial}{\partial v_{\perp}}\right) \tilde{n} \tilde{f}_{0}^{o}=0 \\
& \frac{l_{o}}{\tilde{L}}\left(v_{z} \frac{\partial}{\partial \tilde{z}}+\frac{\partial \tilde{\chi}_{0}}{\partial \tilde{z}} \frac{\partial}{\partial v_{z}}\right) \tilde{f}_{0}^{o} \\
&=\sigma \tau\left[\tilde{n} c\left(\tilde{f}_{0}^{s}, \tilde{f}_{0}^{e}\right)+n_{0}^{i} P\left(\tilde{f}_{0}^{e}\right)\right] .
\end{aligned}
$$

From (51) we conclude that $\tilde{L} / \lambda=O\left(\tilde{n}^{-1}\right)$. We call $\tilde{n}_{*}=\tilde{n}\left(r_{0}=0\right)$ and define $\tilde{L}=\lambda \tilde{n}_{*}^{-1}$.

Now assume $\chi_{p}=O(1)$. We want to derive two important results. First, $\tilde{n} \ll 1$ and therefore, ln $\tilde{n}^{-\beta}>O(1)$; since $\chi_{p}=O(1)$, the electric po- tential does not decrease monotonically from the probe along $\mathbf{B}$ but overshoots to a value $\ln \tilde{n}^{-\beta}$ before decaying to zero at infinity. Second, between the $\tilde{z}$ layer and the probe there is a second inner region.

Suppose that this large overshooting of the potential did not occur and $\chi \leq O(1)$ everywhere. Then, In $\tilde{n}^{-\beta} \leq O(1)$ and therefore, $\tilde{n}=O(1)$. On the other hand, we have $n^{\theta}=O\left(j_{z}^{\theta}\right)$ on the probe surface because all particles have negative $v_{z}$. We found earlier that $j_{z}^{e}$ is conserved to dominant order in the inner layer and is $O(\sigma)$; therefore, $n_{p}^{0} / \tilde{n}=$ $O(\sigma)<O(1)$ ( $n_{p}^{\circ}$ being the electron density at the probe). Let us now multiply Eq. (51) by $v_{z}$ and integrate over v. We obtain

$$
\begin{aligned}
\tilde{n}_{*}\left(\frac{\partial}{\partial \tilde{z}} \int \tilde{f}_{0}^{e} v_{z}^{2} d \mathrm{v}-\frac{\partial \tilde{\chi}_{n}}{\partial \tilde{z}} \int \tilde{f}_{0}^{\theta} d \mathrm{v}\right) & \\
& =n_{0}^{i} \int P\left(\tilde{f}_{0}^{e}\right) v_{z} d \mathrm{v}
\end{aligned}
$$

Since $j_{z}^{e}=O(\sigma)$, the right-hand side is $O\left(n_{0}^{i} \sigma\right)$, negligible compared with the left-hand side. Thus, (52) may be rewritten

$$
\frac{\partial}{\partial \tilde{z}}\left(n^{e} E_{z}^{e}\right)-n^{e} \frac{\partial \chi}{\partial \tilde{z}} \approx 0
$$

where $E_{z}^{e}=\left(n^{e}\right)^{-1} \int f^{e} v_{z}^{2} d \mathbf{v}$. Equation (53) yields

$$
\left.\chi-E_{z}^{e}-\int E_{z}^{o} d\left(\ln n^{\bullet}\right) \approx \text { const (in } \tilde{z}\right) .
$$

From (54) we conclude that condition $n_{p}^{0} / \tilde{n}<O(1)$ seems to be incompatible with the assumption that for all $z, \chi \leq O(1)\left[E_{z}^{a}\right.$ is expected to be $O(1)$ for $\chi \leq O(1)]$. Thus, we shall now assume the ansatz $\tilde{n} \ll 1\left(\ln \tilde{n}^{-\beta} \gg 1\right)$ and shall show that a consistent solution may be obtained.

First, the need for a second inner layer is now easily established. Introduce variables $\tilde{z}$ and $r_{0}$ into (9). To dominant order

$$
\begin{aligned}
& \sigma\left(\mathrm{v}_{\perp} \cdot \frac{\partial}{\partial \mathrm{r}_{\perp 0}}-\frac{1}{\beta} \frac{\partial}{\partial \mathrm{r}_{\perp 0}}\left(\ln \tilde{n}^{-\beta}+\tilde{\chi}_{0}\right) \cdot \frac{\partial}{\partial \mathrm{v}_{\perp}}\right) f_{0}^{i} \\
& \quad+\mu \mathrm{v}_{\perp} \times \hat{\mathrm{z}} \cdot \frac{\partial f_{0}^{i}}{\partial \mathrm{v}_{\perp}}+\sigma \tau \tilde{n}_{*}\left(v_{z} \frac{\partial}{\partial \tilde{z}}-\frac{1}{\beta} \frac{\partial \tilde{\chi}_{0}}{\partial \tilde{z}} \frac{\partial}{\partial v_{z}}\right) f_{0}^{i} \\
& \quad=\sigma \tau\left[c\left(f_{0}^{i}, f_{0}^{i}\right)+\tilde{n} c\left(f_{0}^{i}, f_{0}^{e}\right)\right],
\end{aligned}
$$

where we have again used the coordinates introduced in (37). The right-hand side and the last bracket on the left side of (55) may be dropped because $\tilde{n}_{*} \ll 1$. For $r_{0}>1$, Eq. (37) still holds. By the same argument used to derive (38) we conclude that

$$
f_{0}^{i}=f_{m} \exp \left[-\beta^{-1}\left(\ln \tilde{n}^{-\beta}+\tilde{\chi}_{0}\right)\right] \text {. }
$$


Now introducing $\tilde{z}$ and $r_{0}$ into Eq. (10) we obtain $n_{0}^{i}=\tilde{n} \int \tilde{f}_{0}^{e} d \mathbf{v}$ (quasineutrality) provided that $\epsilon^{2} \ll \tilde{n}_{*}$ (we shall see later that unless an even stronger inequality, $\epsilon^{2} \ll \tilde{n}_{*}^{2}$, is satisfied our whole analysis is invalid). From quasineutrality and Eq. (56) we finally obtain

$$
\tilde{n} \int \tilde{f}_{0}^{e} d \mathrm{v}=\exp \left[-\beta^{-1}\left(\ln \tilde{n}^{-\beta}+\tilde{\chi}_{0}\right)\right]
$$

or $n^{e} \approx \exp \left(-\beta^{-1} \chi\right)$. On the probe, this yields $n_{p}^{e} \approx \exp \left(-\beta^{-1} \chi_{p}\right)$ so that the requirement $n_{p}^{e}=O(\sigma)$ cannot be satisfied since $\chi_{p}=O(1)$. It is obvious, therefore, that a new $z$ layer has to be introduced near the probe. Its characteristic length $\widetilde{\tilde{L}}$ will be such that either the quasineutrality condition or result (56) will break down so that $(57)$ will no longer be true. [Although the value of $\widetilde{\widetilde{L}}$ is not needed for our analysis, we may point out that $\widetilde{L}=R \ll$ $\tilde{L} \equiv \lambda \tilde{n}_{*}^{-1}$, since then the dominant equation for $f^{i}$ will involve $z$ derivatives and the conditions on the probe will affect $f^{i}$ to dominant order. Poisson's equation will yield quasineutrality up to distances to the probe of order of $\epsilon R\left(n^{e}\right)^{-1 / 2}$; the introduction, at such distances, of a third inner layer where charge separation is allowed will not be necessary because a detailed study of the shape of the potential field in the inner layer is not needed for our analysis.]

\section{The Second Inner Layer}

To study the second inner layer we define $\widetilde{z}=z / \widetilde{L}$ and introduce expansions (48) into Eq. (8) to get for $\tilde{f}_{0}^{a}$

$$
\frac{\partial \tilde{f}_{0}^{e}}{\partial \phi}=0,\left(v_{z} \frac{\partial}{\partial \widetilde{\widetilde{Z}}}+\frac{\partial \tilde{\chi}_{0}}{\partial \widetilde{\widetilde{\tilde{z}}}} \frac{\partial}{\partial v_{z}}\right) \tilde{f}_{0}^{a}=0
$$

(note that $\widetilde{L} \ll \lambda \tilde{n}_{*}^{-1}$, which is the effective collision length). The solution to (58) is

$$
\begin{aligned}
\tilde{f}_{0}^{s}=g\left(v_{z}^{2}-2 \tilde{\chi}_{0}, v_{\perp}^{2}\right) h\left[2 ^ { 1 / 2 } \left(\ln \tilde{n}^{-\beta}\right.\right. \\
\left.\left.+\tilde{\chi}_{0}-\chi_{p}\right)^{1 / 2}-v_{z}\right],
\end{aligned}
$$

where $g$ is an arbitrary function and $h$ is the unit step function (with values 1 and 0 for positive and negative arguments, respectively). This solution satisfies (58) and both conditions in (14). [Observe that $d h / d u \equiv \delta(u)$ and $u \delta(u) \equiv 0$ for all $u$; in this note only, $\delta$ is Dirac's function].

If $g$ is known, the electron flux reaching the probe along $\mathbf{B}$ is easily computed

$$
\begin{aligned}
j_{z}^{e} \text { (on the probe) }= & \tilde{n} \int_{-\infty}^{0} v_{z} d v_{z} \int_{0}^{\infty} v_{\perp} d v_{\perp} \\
& g\left[v_{z}^{2}-2\left(\chi_{p}-\ln \tilde{n}^{-\beta}\right), v_{\perp}^{2}\right]
\end{aligned}
$$

(note that on the probe $\chi_{0}=\chi_{p}-\ln \tilde{n}^{-\beta}$ ). To determine $g$ the following argument may be used: Eq. (58) is a particular case of (51) where the gradients in the $z$ direction become large and collisions may be neglected. Thus (51) is valid in both inner layers, where the electrons are strongly repelled by the probe surface because $\ln \tilde{n}^{-\beta}-\chi_{p}>O(1)$. If $\left(\ln \tilde{n}^{-\beta}-\chi_{p}\right) \rightarrow \infty$, no electrons would reach the probe which would then be a perfectly reflecting boundary. With this boundary condition and the condition for $\tilde{z} \rightarrow \infty$ ( $f^{\circ}$ should become Maxwellian to dominant order as results from matching to the outer solution), the solution to (51) would be a Maxwell-Boltzmann distribution throughout both inner layers. [This conclusion may be reached through the same argument used to derive (38).] From the continuity of the solution on the boundary conditions, we expect that for large but finite $\ln \tilde{n}^{-\beta}-\chi_{p}$ only the positive $v_{z}$ tail of the distribution function will differ, to dominant order, from the Maxwell-Boltzmann value; therefore,

$$
g=\exp \left(\tilde{\chi}_{0}\right) f_{m}
$$

Far from the probe where $\tilde{\chi}_{0}+\ln \tilde{n}^{-\beta}-\chi_{p} \gg 1$, Eqs. (59) and (61) yield approximately

$$
\int \tilde{f}_{0}^{e} d \mathrm{v} \approx \exp \left(\tilde{x}_{0}\right)
$$

This result is in conflict with Eq. (57), found to be valid inside the $\tilde{z}$ layer, unless $\tilde{\chi}_{0} \equiv 0$ in this layer.

We can arrive at this result in another way. Note that $\tilde{\chi}_{0}(\tilde{z} \rightarrow \infty)=0$ [see (48) and (49)]. We want to show that $\tilde{\chi}_{0}(\tilde{z} \rightarrow 0)$ vanishes too. The right-hand side of Eq. (52) may be dropped if $\sigma / \tilde{n} \ll 1$; we shall see later that this condition is indeed satisfied. Introducing (57) into (52) and integrating over $\tilde{z}$ we get

$$
\left.\int \tilde{f}_{0}^{e} v_{z}^{2} d \mathrm{v}+\beta \int \tilde{f}_{0}^{o} d \mathrm{v}=\text { const (in } \tilde{\mathbf{z}}\right) .
$$

The constant may be found by evaluating the lefthand side of (63) in the limit $z \rightarrow \infty$, which is known from the outer solution. We find that the constant is $(1+\beta)$. Call $\tilde{\chi}_{0}(\tilde{z} \rightarrow 0)=\chi_{*}$. We now evaluate the left side of (63) in the limit $\tilde{z} \rightarrow 0$, i.e., $\tilde{z} \rightarrow \infty$. Using (59) and (61), (63) yields

$$
\begin{aligned}
& \exp \left(-\beta^{-1} \chi_{*}\right) \\
& \cdot\left(1+\beta-\frac{2 \tilde{n}^{\beta} \exp \left(\chi_{p}-\chi_{*}\right)\left(\ln \tilde{n}^{-\beta}+\chi_{*}-\chi_{p}\right)^{1 / 2}}{\pi^{1 / 2}\left[1+\operatorname{erf} 2^{1 / 2}\left(\ln \tilde{n}^{-\beta}+\chi_{*}-\chi_{p}\right)^{1 / 2}\right]}\right) \\
& =1+\beta
\end{aligned}
$$

and therefore $\chi_{*} \approx 0$, because $\tilde{n}^{\beta} \exp \left(\chi_{p}\right) \ll 1$. 
The result $\tilde{\chi}_{0}=0$ for both $\tilde{z} \rightarrow 0$ and $\tilde{z} \rightarrow \infty$ supports our conclusion following $(61)$ that $\tilde{\chi}_{0}(\tilde{z}) \equiv 0$.

Thus, $\chi \approx \ln \tilde{n}^{-\beta}$ throughout the $\tilde{z}$ layer: The potential grows in the $\widetilde{z}$ layer from $\chi_{p}$ to $\ln \tilde{n}^{-\beta}$, stays constant in $\tilde{z}$ (for a given $r_{0}$ ) and then decays to zero in the outer region. The only dominant phenomenon occurring in the $\tilde{z}$ layer is a modification of $\tilde{f}^{e}$ from its value at $\tilde{z} \rightarrow 0$ [a Maxwellian distribution with a cutoff at $v_{z}=2^{1 / 2}\left(\ln \tilde{n}^{-\beta}-\chi_{p}\right)^{1 / 2}$ ] to its limiting value as $\tilde{z} \rightarrow \infty$ (a complete Maxwellian plus a small, current-carrying distribution, $\delta_{1 c} f_{1 c}$, as results from matching to the outer solution to the first two orders). In Appendix B an analysis of (50) and (51) is given and their solution is matched to the outer solution.

\section{The Determination of the Electron Current}

On the probe we have $g=\exp \left(\chi_{n}\right) \tilde{n}^{\beta} f_{m}$ [see (61)]. If this value is introduced into $(60)$, we obtain

$$
j_{z}^{e}=-(2 \pi)^{-1 / 2} \exp \left(\chi_{p}\right) \tilde{n}^{\beta+1} .
$$

When this result is set equal to (44) and use is made of (49) (remember that $\left.\psi=2 \chi_{0} / \beta\right)$, there results

$p \exp (-s \psi)=-\frac{\partial \psi}{\partial y}, \quad\left(r_{0} \leq 1, y=0\right)$,

where $s=(\beta+1) / 2$ and $p=\exp \left(\chi_{p}\right) \sigma^{-1}(\beta+1)^{-1}$ $\cdot(3 / 16 \gamma)^{1 / 2}$. Equation (66) is the relation we looked for [see (47)]. When Eq. (43) is solved together with the boundary conditions (45), (46), and (66) we may determine the electron flux reaching the probe along B. Since $j_{r}^{e} / j_{z}^{0}=O(\sigma \tau)$, to compute the electron current to this order we may neglect $j_{r}^{\circ}$.

It is now important to determine how accurate our result is for $j_{z}^{e}$ on the probe. We first need to establish the accuracy of our outer solution. Note that $\delta_{1 c} f_{1 \mathrm{c}}^{e} / f_{0}^{e}=O(\sigma / \tilde{n})$ for small $z_{0}$. Thus, $\sigma / \tilde{n}<O(1)$ is a condition necessary for our outer solution to be valid, since otherwise the expansion (23a) breaks down. From (66) we see that $\tilde{n}=O\left[\left(\sigma \ln \sigma^{-1}\right)^{1 /(\beta+1)}\right]$ and therefore,

$$
\sigma / \tilde{n}=O\left[\sigma^{\beta /(\beta+1)}\left(\ln \sigma^{-1}\right)^{-1 /(\beta+1)}\right]<O(1) .
$$

Next, we see from (27) that we must now consider the ratio $\left(-\sigma \sin \phi D_{r_{0}} f_{0}^{e}\right) / f_{0}^{e}$. This ratio is $O(\sigma)$ and thus smaller than $\sigma / \tilde{n}$, except where large radial gradients exist, since then $\partial / \partial r_{0}$ may be large and not $O(1)$. The largest radial gradients will occur at $z_{0} \approx 0, r_{0} \approx 1$. From an analysis of (43) we conclude that $\partial / \partial r_{0} \leq O\left(\tilde{n}^{-1 / 2}\right)$, and thus the ratio considered above will at most be $O\left(\sigma / \tilde{n}^{1 / 2}\right)[<O(\sigma / \tilde{n})]$.

Thirdly, when the analysis of Eq. (20) is carried to third order (we presented the analysis to second order) it is found that both $\delta_{2} j_{z 2}^{e} / \delta_{1} j_{z_{1}}^{e}$ and $\delta_{3} j_{r_{3}}^{a} / \delta_{2} j_{r_{2}}^{e}$ are of the order of $\sigma / \tilde{n}, \delta_{1}^{2} / \tilde{n}$, or $\Delta_{1}$, whichever is the lowest. From Poisson's equation $\delta_{1}^{i} / \tilde{n}=O\left(\epsilon^{2} / \tilde{n}^{2}\right)$ and from the analysis of Appendix B, $\Delta_{1}=\sigma / \tilde{n}_{*}$.

Finally, we may easily determine that our result for $f_{0}^{2}$, Eq. (38), is correct to $O(\sigma \tau)$ while Poisson's equation yields that the outer layer is quasineutral to $O\left(\epsilon^{2} / \tilde{n}^{2}\right)$ [remember that $\partial / \partial r_{0} \leq O\left(\tilde{n}^{-1 / 2}\right)$ ]. Therefore, we conclude that $j_{z}^{\circ}$ is found correctly in the outer layer to order $\sigma / \tilde{n}$ or $\epsilon^{2} / \tilde{n}^{2}$, whichever is the lowest. Now, since $\tilde{L} / L_{0}=\sigma / \tilde{n}_{*}$, we see that in the inner layers $j_{z}^{e}$ is conserved to $O(\sigma / \tilde{n})$ and therefore, the solution to the system (43), (45), (46), and (66) for $x_{p}=O(1)$, will yield the electron current to the probe to $O(\sigma / \tilde{n})$ or $O\left(\epsilon^{2} / \tilde{n}^{2}\right)$, whichever is the lowest; the value of $O(\tilde{n})$ has been given above. Note that the requirement $\epsilon^{2} \ll \tilde{n}^{2}$ implies $\lambda_{D}^{2} \ll l_{\mathrm{e}}^{2}\left(\ln \sigma^{-1} / \sigma^{\beta}\right)^{2 /(\beta+1)}$.

\section{E. The $\left|x_{p}\right| \gg 1$ Case}

Let us now study the case of large $\left|\chi_{p}\right|$. Consider Eq. (66) and take $\chi_{p}$ large and negative. When $x_{p}<-\ln \sigma^{-1}, \psi(y=0)$ becomes small and therefore, $\tilde{n}$ increases to a value near 1 . All this means that the top of the potential hill, $\ln \tilde{n}^{-\beta}$, is now small and that the whole outer layer is only slightly perturbed by the probe. We now have $\tilde{L}=\lambda$. In the second inner layer the electron density may decay to its small value on the probe because we still have $\ln \tilde{n}^{-\beta}-\chi_{p} \gg 1$, although this may now be simply written $-\chi_{p} \gg 1$. The entire analysis given in this section for $\chi_{p}=O(1)$ remains valid. The physical effects accompanying the decrease of $\chi_{p}$ may be briefly described as follows: As $\chi_{p}$ decreases, the top of the potential hill, $\ln \tilde{n}^{-\beta}$, decreases but the difference between the values of the potential field at $z_{0}=0$ and $\widetilde{z}=0$ (the probe surface), $\ln \tilde{n}^{-\beta}-\chi_{p}$, increases indefinitely, so that less and less electrons are collected by the probe.

The case $\chi_{p}$ large and positive is exactly the opposite. As $\chi_{p}$ increases, $\ln \tilde{n}^{-\beta}$ increases but the overshoot, $\ln \tilde{n}^{-\beta}-\chi_{p}$, decreases, as we shall see in the next section. The analysis of the inner layers breaks down for zero overshoot. Thus, there is a maximum (positive) value of $\chi_{p}$ for which our theory is valid. This value is found in the next section to be $\chi_{p} \approx \ln \sigma^{-\beta}$. This result may also be reached by noticing that $\sigma / \tilde{n}$ becomes $O(1)$ for this value and therefore, the analysis of the outer layer breaks down too.

Finally, since the errors in our analysis are $O(\sigma / \tilde{n})$ and $O\left(\epsilon^{2} / \tilde{n}^{2}\right)$ and since $\tilde{n}$ decreases as $\chi_{p}$ grows, the theory becomes less accurate as $\chi_{p}$ grows. Since the 
right-hand side of (66) is large for large $\psi$, the results may be accepted right up to $\chi_{p} \approx \ln \sigma^{-\hat{\beta}}$.

\section{THE PROBE CHARACTERISTIC}

The net current to the probe is

$$
I=I^{e}-I^{i}=e \int_{s} J^{e} d s-Z_{i} e \int_{s} J^{i} d s,
$$

where the integrals are over the probe surface and $J^{a}$ is the $a$ species particle flux incident on the probe.

As seen in Sec. III the ratio of electron flux across $\mathrm{B}$ to electron flux along $\mathrm{B}$ is $O(\sigma \tau)$. To this order we may obtain $I^{e}$ by integrating the $z$ component of the electron flux over the probe cross section:

$$
\frac{I^{e}}{I_{\infty}^{e}}=-(2 \pi)^{1 / 2} \sigma \int_{0}^{1} j_{z 1}^{e}(x, y=0) x d x,
$$

where $I_{\infty}^{\circ}=4 \pi R^{2} e N_{o \infty}\left(k T_{o} / m_{\theta}\right)^{1 / 2}(2 \pi)^{-1 / 2}$ and is the random current collected by a spherical probe in the absence of electric and magnetic fields; $\sigma j_{z 1}^{*}$ may be obtained from (44) after Eq. (43), together with the boundary conditions (45), (46), and (66), have been solved.

If $\chi_{p}$ is large and negative, Eqs. (43) and (66) may be linearized because $\psi$ is small everywhere. The resulting linear equation for $\psi$ may easily be solved ${ }^{20}$ to give $\psi(x<1, y=0)=2 \pi^{-1} p E(x)$ where $E$ is the complete elliptic integral of the second kind. From (44) and (66) for small $\psi$ we obtain

$$
j_{z 1}^{e} \approx-(\beta+1)(8 \gamma / 3 \pi)^{1 / 2} p(1-\mathrm{s} \psi)
$$

and finally

$$
\frac{I^{e}}{I_{\infty}^{e}} \approx \frac{\exp \left(\chi_{p}\right)}{2}\left(1-\frac{\exp \left(\chi_{n}\right)}{\pi \sigma(3 \gamma)^{1 / 2}}\right) .
$$

We see that this analytical result ceases to be valid for $\chi_{p} \gtrsim-\ln \sigma^{-1}$. Note that there is no $\beta$ dependence for large, negative $\chi_{p}$. Also, we get $I^{e} \sim \exp \left(\chi_{p}\right)$ only for very large, negative $\chi_{p}$. The factor $\frac{1}{2}$ is due to the channel effect of $B$ : the effective collecting area is twice the probe cross section, i.e., half the probe area.

For $\chi_{p} \gtrsim-\ln \sigma^{-1}$, Eqs. (43), (45), (46), and (66) have to be solved numerically. Some information, however, may still be obtained analytically. In an average, approximate sense we may neglect the $x$ dependence in condition (66). Call $\psi^{*}=\psi(x<1$, $y=0)$ and $\psi_{y}^{*}=\partial \psi / \partial y(a<1, y=0)$. For a given $\psi^{*}$, Eqs. (43), (45), and (46) give $\psi_{v}^{*}$; thus, $\psi_{y}^{*}$ is a function of $\psi^{*}$ and we write $\psi_{v}^{*}=-G\left(\psi^{*}\right)$. Obviously, $G(0)=0$ and $d G / d \psi^{*} \geq 0$ for all $\psi^{*}$. From (44), (66), and (68) we then obtain

\footnotetext{
${ }^{20} \mathrm{~J}$. R. Sanmartin, Ph.D. thesis, University of Colorado (1967)
}

$$
\begin{aligned}
\frac{I^{e}}{I_{\infty}^{e}} & \approx 2^{-1} \exp \left(\chi_{p}\right) \exp \left(-s \psi^{*}\right) \\
& \approx 2\left(\frac{\gamma}{3}\right)^{1 / 2} \sigma(\beta+1) G\left(\psi^{*}\right)
\end{aligned}
$$

and an implicit equation for $I^{\theta} / I_{\infty}^{*}$ may be derived

$$
\frac{I^{e}}{I_{\infty}^{e}} \approx 2\left(\frac{\gamma}{3}\right)^{1 / 2} \sigma(\beta+1) G\left(\frac{\chi_{p}-\ln 2 I^{e} / I_{\infty}^{o}}{s}\right) .
$$

For $\chi_{p}$ large and negative, $\psi^{*}$ is small and therefore, $G\left(\psi^{*}\right)$ is linear in $\psi^{*}, G=\psi^{*} d G / d \psi^{*}$. There results

$$
\frac{(3 / 8)^{1 / 2}}{4 \sigma d G / d \psi^{*}} \frac{I^{e}}{I_{\infty}^{e}}+\ln \frac{I^{e}}{I_{\infty}^{\theta}}+\ln 2 \approx \chi_{p} .
$$

As noticed before, $\beta$ disappears from the $C-V$ diagram in this range of $\chi_{p}$. For $\chi_{p}<-\ln \sigma^{-1},(71)$ gives

$$
\frac{I^{e}}{I_{\infty}^{e}} \approx \frac{\exp \left(\chi_{p}\right)}{2}\left(1-\frac{(3 / \gamma)^{1 / 2} \exp \left(\chi_{p}\right)}{8 \sigma d G / d \psi^{*}}\right) .
$$

Comparing (72) to (69) we get $d G / d \psi^{*} \approx 3 \pi / 8$.

If $G$ were linear for all $\psi^{*}$, for $\chi_{p} \gg \ln \sigma^{-1}(71)$ would give

$$
\frac{I^{e}}{I_{\infty}^{e}} \approx \sigma \frac{4 d G / d \psi^{*}}{(3 / \gamma)^{1 / 2}}\left(\chi_{p}-\ln \chi_{\nu}\right)
$$

with a limiting slope of $O(\sigma)$, very small; thus, quasisaturation would result. Actually, $G$ will not be linear and $d G / d \psi^{*}$ is expected to decay for large $\psi^{*}$ with the consequence that the saturation would be sharper; in general, we expect $d G / d \psi^{*} \leq G / \psi^{*}$.

Unfortunately, the formulation of Sec. III breaks down at $\chi_{p} \approx \ln \sigma^{-\beta}$ because then $\sigma / \tilde{n}_{*}$ is $O(1)$. The breakdown may be seen in another way. $\mathrm{Ob}$ serve that, from (66), $d \psi^{*} / d \chi_{p}>0$ and therefore, $d G / d \chi_{p}>0$. Then, also from $(66), d / d \chi_{p}\left(\chi_{p}-\right.$ $\left.s \psi^{*}\right)>0$ or

$$
\frac{d \beta \psi^{*} / 2}{d \chi_{p}}<\frac{\beta}{\beta+1}<1 ;
$$

$\beta \psi^{*} / 2$ is the value of $\chi$ at the top of the potential hill. The inequality (73) means that $\chi_{p}$ increases faster than and finally catches up with $\beta \psi^{*} / 2$, and then the analysis of the inner layers breaks down. The condition of no overshoot, $x_{p} \approx \beta \psi^{*} / 2$, implies from (66)

$$
\chi_{p} \approx-\beta \ln \sigma+O(1)
$$

In (73) we see that the overshoot only remains for cold electrons $(\beta \rightarrow \infty)$ in the limit $\chi_{p} \rightarrow+\infty$.

Nonetheless, it is possible that for small enough $\sigma$, a saturation appears in $I^{\theta}$ before $\chi_{p} \approx \ln \sigma^{-\beta}$. This is because an analysis of Eq. (43) seems to indicate that $d G / d \psi^{*}$ will decay quite fast for large $\psi^{*}$. 
Equation (43) was solved using the University of Colorado CDC 3600 computer and then the integral in (68) was calculated. To bring condition (45) to a finite boundary, the behavior of $\psi$ at infinity was found analytically using a coordinate asymptotic $\operatorname{expansion}^{20}$

$\psi \approx \frac{a_{0}}{\rho}+a_{0}^{2} \cos ^{2} \frac{\eta}{\rho^{2}}+O\left(\rho^{-3}\right)$, as $\rho \rightarrow \infty$,

where $\rho=\left(x^{2}+y^{2}\right)^{1 / 2}$ and $\operatorname{tn} \eta=y / x$. It is interesting to note that a volume integration of (43) results in the relation

$$
a_{0}+\left.\int_{0}^{1} x d x \frac{\partial \psi}{\partial y}\right|_{y=0}=0
$$

An over-relaxation factor could have been derived from (75) to accelerate the numerical convergence. \{Equation (75) can be understood by rewriting (43) as

$$
\nabla^{2} \psi=x^{-1} \frac{\partial}{\partial x} x \frac{\partial}{\partial x}[\exp (-\psi)+\psi]
$$

This is a Poisson-like equation. The volume integral of the right-hand side, which represents the space charge, vanishes and therefore, $a_{0}$ in (74) should be equal to the total charge on the disc $(x<1, y=0)$, as given in (75).

In Fig. 1 we give $(8 \pi)^{1 / 2} I^{e} / I_{\infty}^{e}$ (writing $T_{e}$ and $N_{\infty}$ for $T_{e \infty}$ and $N_{c \infty}$ ) as a function of $\chi_{p}$ and $\sigma$, for $\beta=1$ (and $Z_{i}=1$ ). The variation with $\beta$ is not given, and we only present negative or slightly positive values of $\chi_{p}$. This is because when the computations were made the matching of the inner and outer $\approx$ layers was not correct and an additional factor $2[1+$ erf $\left.\left(\beta \psi / 2-\chi_{p}\right)^{1 / 2}\right]^{-1}$ appeared on the left-hand side of (66). This factor caused $\partial / \partial \beta\left(I^{e} / I_{\infty}^{e}\right)$ to be negative while we may easily derive from (66) the result

$$
\frac{\partial}{\partial \beta} \frac{I^{e}}{I_{\infty}^{*}} \approx \frac{I^{e}}{2 I_{\infty}^{e}} \frac{G-\psi^{*} d G / d \psi^{*}}{2^{-1}(\beta+1) G+d G / d \psi^{*}}>0 .
$$

The same factor made $\partial^{2} / \partial \chi_{p}^{2} I^{0} / I_{\infty}^{e}$ become large and positive when $\chi_{p}$ approached $\beta \psi^{*}\left(\chi_{n}\right) / 2$. Thus, the numerical results obtained were valid only for $\chi_{p}$ such that erf $\left(\beta \psi / 2-\chi_{p}\right)^{1 / 2} \approx 1$ although the theory is valid up to $\chi_{p} \approx \ln \sigma^{-\beta}$. Therefore, it was not possible to determine whether electron saturation sets in for moderate $\chi_{p}$.

To complete the theoretical description of the $C-V$ diagram we need $I^{i}$. From now on we shall use conditions $\left(\mathrm{D}^{\prime}\right)$ and $\left(\mathrm{E}^{\prime}\right)$ instead of $(\mathrm{D})$ and $(\mathrm{E})$. We consider two distinct regions in the $C-V$ diagram.

The first region covers large, negative $\chi_{p}$. How large $\chi_{p}$ has to be will be indicated below. We want to show that in this region the effect of $\mathrm{B}$ on $I^{i}$ may

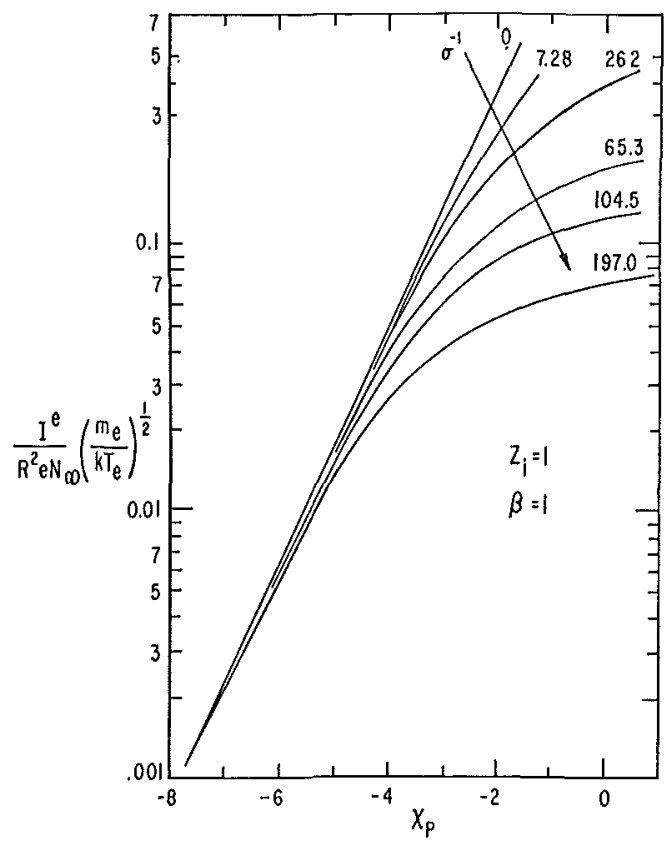

Fra. 1. The electron current as a function of $\chi_{p}$ and $\sigma$ (the ratio of electron cyclotron radius to probe radius).

be neglected. We shall then use known results ${ }^{2-11}$ for the ion current for $B=0$. Assume $B=0$ and first take the collisionless limit $\lambda \gg R$ in condition $\left(\mathrm{D}^{\prime}\right)$. Then, we refer the reader to Figs. 20,21, 27b, and 39 in Ref. 5. We see that for $\beta=O(1), \epsilon<O(1)$ [our conditions $(\mathrm{A})$ and $(\mathrm{C})]$ and large, negative $\chi_{p}$ (approximately for $\epsilon<0.1$ and $\chi_{p}<-3.5$ ) a change of $O(1)$ in $\chi_{p}$ produces a relative variation in $I^{i}$ which is of order of $10^{-1}$; a substantial change in the form of $f^{i}$ at infinity (a Maxwellian or a monoenergetic distribution in either spherical or cylindrical coordinates) or in the shape of the probe (sphere or cylinder) produces similar weak relative variations in $I^{i}$; and the use of the simplifying assumption $n^{e} \approx \exp (\chi)$ (which means that the probe completely repels the electrons) produces no noticeable effects. For small changes in all these variables or conditions, the relative variations in $I^{\boldsymbol{i}}$ will be of a higher order.

Now assume that $\chi_{p}<-\ln \sigma^{-1}$. For such $\chi_{p}$ the effect of $\mathrm{B}$ on $I^{e}$ is small, except for the factor $\frac{1}{2}$ due to the channeling effect [see (69)]. The outer region is only slightly disturbed by the probe $(\tilde{n} \approx 1)$. The problem of ion collection may then be restricted to a study of the inner layers. The situation is not the same as for $B=0$, but the differences are small: a slight effective decrease in $\chi_{p}$ (due to the small overshooting) and a slight distortion of the spherical symmetry which amounts to a small change in both the shape of the probe and the form of $f^{i}$ at infinity 
in the inner layers. We still have $n^{e} \approx(\exp \chi)$. Therefore, in agreement with the analysis of the preceding paragraph, we conclude that the effect of $\mathrm{B}$ on $I^{i}$ will be small compared with the effect on $I^{c}$, itself small for such $\chi_{v}$. [Although the dynamics of the ions are not the same for both $B=0$ and $B \neq 0$, this is not expected to yield noticeable effects if $\epsilon \ll 1$ and $l_{i}>R$, conditions $(\mathrm{C})$ and $\left(\mathrm{E}^{\prime}\right)$. The dynamics for spherical and cylindrical probes are also different and yet the results for $I^{i}$, for the same collecting area, are quite similar if $\epsilon \ll 1 .^{5}$ Also, the analysis of Ref. 3 for $\epsilon \ll 1$ shows that for large, negative $\chi_{p}$, $I^{i}$ only depends on the structure of the nonquasineutral sheath and on the location of the end of the sheath, very close to the probe surface, and thus is insensitive to $\mathrm{B}$ if $l_{i}>R$.]

Now, when the case $\lambda>R$ instead of $\lambda \gg R$ is considered, the reader is referred to Fig. 6 of Ref. 11 . It is shown there that the ion saturation current is nearly the same for all values of $\lambda / R$ above $\lambda / R=10$ Since this result was given for weakly ionized gases, we should expect that for fully ionized gases (for which the effective mean free path near the probe is larger than $\lambda$, its value at infinity, because both $n^{e}$ and $n^{i}$ decrease there) the saturation current should be insensitive to the ratio $\lambda / R$, down to values very close to $\lambda / R=1$. We may extend this conclusion to large but finite, negative $\chi_{p}$ because in Ref. 9, Fig. 11 , it is shown that for $\epsilon \ll 1$ and such $\chi_{p}, d I^{i} / d \chi_{p}$ is small. Thus, in conclusion, for $\chi_{p}$ less than both $\ln \sigma^{-1}$ and -3.5 , we may use the results for $I^{i}$ given in Ref. 5 , if $l_{i}>R, \lambda>R$. For $I^{c}$ we may use (69). Since $\left(\mathrm{E}^{\prime}\right)$ implies $\mu<\sigma$, the floating potential is included in this region of the $C-V$ diagram.

A second region of the $C-V$ diagram may now be considered. It corresponds to values of $\chi_{p}$ for which $I^{i} \ll I^{i}$. Then, $I^{i}$ may be neglected and the numerical results of Fig. 1 for $I^{e}$ are valid for the net current $I$. Typically, $I^{i} \ll I^{e}$ for $\chi_{p}>-2$. [Although $I^{e}$ is substantially decreased by B we expect that $I^{i} / I^{e}=$ $O(\mu)$ for such $\chi_{p}$, as is the case when $B=0$. The reason is that $I^{e}$ is decreased because few electrons reach the probe and since quasineutrality is maintained up to a short distance from the probe, few ions reach it too. Thus, $I^{i} / I^{0}$ will be approximately of the order of the square root of the thermal velocity ratio, i.e., $O(\mu)$.] Since $x_{p}$ must be less than $\ln \sigma^{-\beta}$ because otherwise our theory is not valid, the typical range of this second region is $-2<\chi_{p}<\ln \sigma^{-\beta}$.

\section{DISCUSSION}

In this paper we present an asymptotic analysis of electron collection by a spherical probe in the pres- ence of a strong magnetic field B. The analysis is based on the assumptions that the ion-to-electron temperature ratio is $O(1)$, that both the electron cyclotron radius $l_{s}$ and Debye length $\lambda_{D}$ are small compared with the probe radius $R$ and that $R$ is comparable to or smaller than the mean free path $\lambda$ and the ion cyclotron radius $l_{i}$.

Under these conditions, it is found that, for a probe potential comparable to or smaller than the electron kinetic energy, ${ }^{21}$ three distinct regions may be distinguished around the probe. Far away there is a collision-dominated, quasineutral, outer region where the ions have a Maxwell-Boltzmann distribution and electrons are drawn by collisions into the "shadow" (the magnetic tube of force impinging on the probe). Inside the shadow and near the probe there is a first inner layer, still quasineutral, where the electric potential does not change in the direction of $\mathrm{B}$ and is positive and much larger than the potential on the probe. Close to the probe there is a second collisionless, inner layer where the potential decays to its value on the probe. The description of the electrons in the inner layers is essentially one-dimensional, and thus there is no need for a detailed description of the shape of the potential inside these layers. On the other hand, the electric field is not confined to a sheath but penetrates the outer region where it controls the diffusion process. In this outer region the probe appears as a thin disc and its shape along B is not important.

The overshooting of the potential, i.e., its growth from the value on the probe to a large value in the intermediate layer before decaying to zero in the outer region, may be explained as follows. Electron flow across $B$ is strongly inhibited by the magnetic field. Therefore, as a consequence of mass conservation, the electron flux along $B$ remains constant over long distances. On the other hand, friction with the ions tries to damp such a flux. As a compromise, the electron flux along $B$ varies slowly in the direction of the magnetic field, but has always a small value (in nondimensional units). Since there are no outgoing particles on the probe surface, we conclude that the electron density has to be very small there. The potential overshoot is built up to allow such low electron density (even if the probe potential is positive) near the probe.

When the probe potential becomes large and negative, the overshoot disappears in agreement with the explanation just given. When the probe

${ }^{21}$ We take the space potential as the origin of potentials. The discussion now given is valid for either positive or negative probe potentials. 
potential becomes large and positive, the value of the potential in the intermediate layer grows too but slowly; eventually, the overshoot disappears and then our theory breaks down. As a consequence of the overshoot the usual sharp knee in the $C-V$ diagram at space potential becomes blurred, in agreement with experimental evidence. (Notice that the value of the potential in the first inner layer, which varies with the potential applied to the probe, acts as an effective space potential for the inner layers.)

Although the theory has been based on the assumption $l_{\theta} / R \ll 1$, the following point should be noticed now. Since the shape of the probe along $B$ is unimportant, our results are still valid if we consider a thin disk perpendicular to B instead of a sphere. If we now go back to the linear analysis of Sec. IV, valid for large, negative potentials, and consider increasing values of $l_{e} / R$, up to the limit $l_{e} / R \rightarrow \infty$ $(B \rightarrow 0)$, we see that the known result for the collection of a repelled species in the absence of a magnetic field is recovered, while the validity of the theory becomes limited to negative potentials since the overshoot goes to zero as $l_{\theta} / R$ increases. Thus, our results would appear to be approximately valid (for a restricted potential range) for weak magnetic fields too. We also see that an effect of the overshoot is to extend to positive potentials, the simple, known result for the repelled species current.

The present theory can be easily extended to cover weakly ionized gases. The diffusion equation of the outer region would now be linear. If we compare our analysis with the Bohm-Sugawara theory, ${ }^{16,17}$ the following differences may be noted. First, their theory is not asymptotic and thus there is no way of determining the errors or improving the approximation. Second, their results are only useful for zero ion temperature; they did not find the phenomenon of potential overshooting and its effect on the $C-V$ diagram. Third, because of the absence of overshoot the matching of inner and outer regions could not be made properly and their results are quantitatively crude. Finally, notice that while we matched the inner and outer regions locally at every point of the probe cross section, they solved the diffusion equation in the outer region using a global, incorrect matching.

The basic drawback of our theory lies in its exclusion of anomalous transport processes. As a consequence, the probe perturbs the plasma over a long distance along $\mathbf{B}$ (of order of $\lambda R / l_{c}$ ). On the other hand, the current drawn from the plasma is very small. Moreover, if our conditions are satisfied, the results are correct within known errors. The error of the numerical computations is $2 \%$. The error in the transport coefficients is known to be 5 or $10 \%$. (In Sec. III the use of an average for the usual Coulomb logarithm, impairs the accuracy slightly, the smaller $l_{o} / R$ the stronger the impairment. However for $l_{\sigma} / R$ as small as 0.0005 , the error is still 15\%.) Finally, the errors due to the asymptotic analysis are of order $\left(l_{e} / R\right)(\tilde{n})^{-1}$ and $\left(\lambda_{D} / R\right)^{2}(\tilde{n})^{-2}$ where $O(\tilde{n})$ is given at the end of Sec. III.

\section{ACKNOWLEDGMENTS}

The author wishes to express his appreciation to Professor C. Forbes Dewey for helpful discussions and guidance.

The author acknowledges support from the Advanced Research Projects Agency of the Department of Defense, monitored by Army Research Office-Durham under Contract DA-31-124-ARO (D)-139 and the United States Air Force (AFSC) under Contract F33615-67-C-185. Parts of this work were performed under auspices of the United States Atomic Energy Commission, Contract No. AT (30-1)-1238.

\section{APPENDIX A}

In this Appendix we want to prove first that Eq. (26) for $f_{0}^{e}$ has a unique solution an isotropic Maxwellian distribution. While physically apparent, it can be proved simply. Define $H=\int d \mathbf{v} f_{0}^{a} \ln f_{0}^{a}$, introduce $\partial f_{0}^{e} / \partial t$ in the right-hand side of $(26)$ and multiply by $\left(1+\ln f_{0}^{e}\right)$. Then,

$$
\begin{aligned}
& \frac{d H}{d t}=\int d \mathrm{v}\left(\ln f_{0}^{e}+1\right) c\left(f_{0}^{e}, f_{0}^{e}\right) \\
& \quad+n^{i} \int d \mathrm{v}\left(\ln f_{0}^{e}+1\right) P\left(f_{0}^{e}\right) \equiv \frac{d H_{1}}{d t}+\frac{d H_{2}}{d t}
\end{aligned}
$$

The usual $H$ theorem gives $d H_{1} / d t \leq 0$. Since

$$
P\left(f_{0}^{e}\right)=\frac{A}{|v|^{3}} \frac{\partial}{\partial \nu}\left(1-\nu^{2}\right) \frac{\partial f_{0}^{e}}{\partial \nu}
$$

$$
\left(A>0, \nu=v_{z} /|\mathbf{v}|, \partial f_{0}^{e} / \partial \phi=0\right) \text { there results }
$$

$$
\begin{aligned}
\frac{d H_{2}}{d t} & =A n^{i} 2 \pi \int_{0}^{\infty} \frac{d|\mathrm{v}|}{|\mathrm{v}|} \int_{-1}^{1} d \nu\left(\ln f_{0}^{e}+1\right) \frac{\partial}{\partial \nu}\left(1-\nu^{2}\right) \frac{\partial f_{0}^{e}}{\partial \nu} \\
& =-2 \pi A n^{i} \int_{0}^{\infty} \frac{d|\mathrm{v}|}{|\mathrm{v}|} \int_{-1}^{1} d \nu\left(1-\nu^{2}\right)\left(\frac{\partial f_{0}^{e}}{\partial \nu}\right)^{2} \frac{1}{f_{0}^{e}} \leq 0 .
\end{aligned}
$$

Both $d H_{1} / d t$ and $d H_{2} / d t$ have to vanish independently for (26) to be satisfied; thus, $\partial f_{0}^{e} / \partial \nu=0$ and $f_{0}^{e}$ is Maxwellian and isotropic.

Second, we want to prove that the ion distribution given in Eq. (38) is the solution to the equation 


$$
\begin{aligned}
\sigma\left(\mathrm{v}_{\perp} \cdot \frac{\partial}{\partial \mathrm{r}_{\perp 0}}-\beta^{-1} \frac{\partial \chi_{0}}{\partial \mathrm{r}_{\perp 0}} \cdot \frac{\partial}{\partial \mathrm{v}_{\perp}}\right) f_{0}^{i}+ & \mu \mathrm{v}_{\perp} \times \hat{\mathrm{z}} \cdot \frac{\partial f_{0}^{i}}{\partial \mathrm{v}_{\perp}} \\
& =\sigma \tau c\left(f_{0}^{i}, f_{0}^{i}\right) .
\end{aligned}
$$

Introduce $\partial f_{0}^{i} / \partial t$ in the left-hand side of (A1) and define $H=\int d \mathbf{r}_{\perp 0} \int d v f_{0}^{i} \ln f_{0}^{i}$. The spatial integration is over a plane perpendicular to $B$, i.e., at a given $z_{0}$, and reaches up to large values of $\left|\mathbf{r}_{\perp 0}\right|$ so that $f_{0}^{i}\left(-\mathrm{v}_{\perp}\right)=f_{0}^{i}\left(\mathrm{v}_{\perp}\right)$. The argument used in Ref. 22 to show that the Maxwell-Boltzmann distribution is the only possible steady distribution function for a gas under the action of a potential field and bounded by perfectly reflecting walls, may be now reproduced with the result given in Eq. (38). An arbitrary $z_{0}$ dependence is left in the density and the average $v_{z}$ velocity, because $z_{0}$ and $v_{z}$ only enter (A1) as parameters. Equation (38) essentially means that boundary effects due to the probe are small so far from the probe.

\section{APPENDIX $\mathbf{B}$}

In this Appendix we discuss briefly the evolution of the electron distribution function in the first inner layer. To dominant order $f^{c}$ is described by Eq. (51) where $\tilde{\chi}_{0}(\tilde{z}) \equiv 0$. We want to point out first that the equation for $\tilde{f}_{0}^{\theta}$ should then read

$\tilde{n}_{*}\left(v_{z} \frac{\partial}{\partial \tilde{z}}+\tilde{\Delta}_{1} \frac{\partial \tilde{\chi}_{1}}{\partial \tilde{z}} \frac{\partial}{\partial v_{z}}\right) \tilde{f}_{0}^{e}=\tilde{n} c\left(\tilde{f}_{0}^{e}, \tilde{f}_{0}^{e}\right)+n_{0}^{i} P\left(\tilde{f}_{0}^{e}\right)$,

where the next term in the expansion of $\chi$ in (48) has been included, although by definition $\tilde{\Delta}_{1} \tilde{\chi}_{1} \ll 1$. Notice that the initial value of $\tilde{f}_{0}^{e}, \tilde{f}_{0}^{e}(\tilde{z}=0)$, is a Maxwellian distribution with a cutoff in velocity space at a large value of $v_{z}$. The diffusion process due to the collision term on the right-hand side of (B1) will smooth this discontinuity progressively. Thus, the region of interest for the evolution of $\tilde{f}_{0}^{a}$ is around this discontinuity where strong nonlinear effects are produced; $\partial \tilde{f}_{0}^{c} / \partial v_{z}$ will be quite large there so that the second term on the left in (B1) is expected to be important although $\tilde{\Delta}_{1} \tilde{\chi}_{1} \ll 1$.

In the limit $\tilde{z} \rightarrow \infty$, the discontinuity will have disappeared and for very large values of $\tilde{z}$ we may write $\tilde{f}_{0}^{o}=f_{0}+f_{1}$, where $f_{1} \ll f_{0}$ and they satisfy

$$
\begin{gathered}
\tilde{n}_{*} v_{z} \frac{\partial f_{0}}{\partial \tilde{z}}=\tilde{n} c\left(f_{0}, f_{0}\right)+n_{0}^{i} P\left(f_{0}\right), \\
\tilde{n}_{*}\left(v_{z} \frac{\partial f_{1}}{\partial \tilde{z}}+\tilde{\Delta}_{1} \frac{\partial \tilde{\chi}_{1}}{\partial \tilde{z}} \frac{\partial f_{0}}{\partial v_{z}}\right) \\
=\tilde{n}\left[c\left(f_{0}, f_{1}\right)+c\left(f_{1}, f_{0}\right)\right]+n_{0}^{i} P\left(f_{1}\right) .
\end{gathered}
$$

${ }^{22} \mathrm{~S}$. Chapman and T. G. Cowling, The Mathematical Theory of Non-Uniform Gases (Cambridge University Press, New York, 1960), Chap. 4.
To match to the outer solution we write $\chi_{0}$ and $f_{0}^{e}$ in inner variables:

$$
\begin{aligned}
& \chi=\chi_{0}\left(\frac{\sigma}{\tilde{n}_{*}} z\right)+\Delta_{1} \chi_{1}\left(\frac{\sigma}{\widetilde{n}_{*}} \tilde{z}\right)+\cdots \\
&= \ln \tilde{n}^{-\beta}+\frac{\sigma}{\widetilde{n}_{*}} S \tilde{z}+\Delta_{1} \chi_{1}(0)+\cdots \\
& f^{e}=\tilde{n} f_{m}-\sigma S\left(\frac{\tilde{n} \tilde{z}}{\tilde{n}_{*} \beta} f_{m}-\frac{\beta+1}{\beta} c^{-1} \frac{\partial f_{m}}{\partial v_{z}}\right) \\
& \quad+\sigma(\beta+1) \frac{\partial \tilde{n}}{\partial r_{0}} \sin \phi \frac{\partial f_{m}}{\partial v_{\perp}}+\cdots,
\end{aligned}
$$

where $S\left(r_{0}\right)=\partial \chi_{0} /\left.\partial z_{0}\right|_{z_{0}=0}$ and $c^{-1} \partial f_{m} / \partial v_{z}$ is such that

$\frac{\partial f_{m}}{\partial v_{z}}=c\left(f_{m}, c^{-1} \frac{\partial f_{m}}{\partial v_{z}}\right)+c\left(c^{-1} \frac{\partial f_{m}}{\partial v_{z}}, f_{m}\right)+P\left(c^{-1} \frac{\partial f_{m}}{\partial v_{z}}\right)$

Matching (B4) to the expansion in (48) we see that

$\tilde{\Delta}_{1} \tilde{\chi}_{1} \approx \sigma / \tilde{n}_{*} S \tilde{z}+\Delta_{1} \chi_{1}(0)$, as $\quad \tilde{z} \rightarrow \infty$.

Introducing this value of $\tilde{\chi}_{1}$ into (B3) we notice that

$f_{0}=f_{m}, \quad f_{1}=-\sigma S\left(\frac{\tilde{z} f_{m}}{\tilde{n}_{*} \beta}-\frac{\beta+1}{\tilde{n} \beta} c^{-1} \frac{\partial f_{m}}{\partial v_{z}}\right)$

is a solution to (B2) and (B3) and remembering the expansion for $f^{e}$ in (48) we see that this solution reproduces (B5) except for the last term; this last term in (B5) is yielded by the solution to (50), $\tilde{\delta}_{1} \tilde{f}_{1}^{\theta}$, as can easily be verified.

Notice that (B1) is parabolic in $\tilde{z}$. Thus, with the value of $\tilde{f}_{0}^{e}(\tilde{z}=0)$ given, the matching of $\tilde{f}_{0}^{o}$ to (B5) cannot be imposed as an additional condition; instead (B7) has to be selected by Eq. (B1) itself. Strong evidence can be presented that this is actually the case. For instance, (B3) also admits as solutions

$$
f_{1}=\frac{\sigma S}{\widetilde{n}_{*}} \tilde{z} f_{m}, \quad f_{1}=\frac{\sigma S}{\tilde{n}} c^{-1} \frac{\partial f_{m}}{\partial v_{z}} .
$$

The first solution is ruled out by (B1) because such $f_{1}$ yields $\int \tilde{f}_{0}^{t} v_{z} d v=0$ while from (B1)

$$
\frac{\partial}{\partial \tilde{z}} \int \tilde{f}_{0}^{e} v_{z} d \mathbf{v}=0
$$

and $\tilde{f}_{0}^{o}(\tilde{z}=0)$ is such that $\int \tilde{f}_{0}^{e}(\tilde{z}=0) v_{z} d \mathbf{v} \neq 0$. The second solution in (B8) is also ruled out because such $f_{1}$ yields $d H_{z} / d z=0$ where $H_{z}=\int v_{z} \tilde{f}_{0}^{e} \ln \tilde{f}_{0}^{c} d v$, while multiplying (B1) by $\left(\ln \tilde{f}_{0}^{e}+1\right)$ and integrating over $\mathrm{v}$ we get

$$
\frac{d H_{z}}{d \tilde{z}}=\int\left(\ln \tilde{f}_{0}^{e}+1\right)\left[\tilde{n} c\left(\tilde{f}_{0}^{e}, \tilde{f}_{0}^{e}\right)+n_{0}^{t} P\left(\tilde{f}_{0}^{o}\right)\right] d v<0
$$

The inequality follows from the usual $H$ theorem and the fact that $\int \tilde{f}_{0}^{e} y_{z} d v \neq 0$ (see Appendix A). 